\title{
Short Term Load Forecasting Solution Methodologies: Literature Review 2013 Survey Paper
}

\author{
M.Arif ${ }^{[1]}$, Tahir Nadeem ${ }^{[2]}$, Haider Ali ${ }^{[3]}$, Syed Wajahat Ali ${ }^{[4]}$, Usman \\ Khalid $^{[5]}$ M. Baseer ${ }^{[6]}$ \\ ${ }^{2}$ (UET,Taxila,Pakistan) \\ 1,3-6 (CIIT Abbottabad,Pakistan)
}

\begin{abstract}
This paper presents the comprehensive study of the solution methodologies used so for the for Short Term Load Forecasting, these methodologies characterized by the methods and models as classical and artificial intelligent techniques. Statistical Technique includes Similar day approach, Linear regression, Time series method,State space and intelligent techniques includes Artificial Neural Network (ANN),SVM(Support Vector Machine Regression), Fuzzy logic, SO(Particle Swarm Optimization),GA(Genetic Algorithm),ACA(Ant Colony Algorithm) and also there are hybrid techniques like Fuzzy and ANN, Adaptive neural fuzzy interface system, LS-SVM Optimized by Bacterial Colony Chemotax is Algorithm and GA based SVM and the last one is the Machine From this survey we show the importance of the short term load forecasting in operation and control and we conclude that the more promising method used for STFL is ANN(Artificial Neural Network) And A novel network Support Vector Machine and Ada Boost Regression techniques has great potential for the short term load forecasting other methods are also used for STFL.
\end{abstract}

Keywords: Artificial Neural network, Artificial intelligence, Regression analysis, Short Term Load Forecasting, Support vector machine.

\section{Introduction}

There is a numerous research literatures on the short term load forecasting published since 1956, but the research in this area is stilled a challenge to the power engineer researchers because of its complexity and nonlinearity model [1]. Now a day's Artificial Intelligence based techniques are more frequently used for the STLF problems [2]. Artificial Neural Network based STLF models are most promising tool used by the researcher but as there are numerous hidden layers in the neural network models making it slow in the training and testing phase [3]. There are numerous ANN flavours used for the STLF [4]. Another novel neural technique which attains a great attention of the researcher is Support Vector Machine [5] which is more fast and accurate than ANN.

Load forecasting is the important step of the power system planning and is critical component of energy management system .Accurately load forecasting helps the electric utility to make the system operation economical i.e. make unit commitment accurately and also the dispatch units economically. In terms of lead time, load forecasting is divided into four categories[6]:

- Long-term forecasting with the lead time of more than one year

- Mid-term forecasting with the lead time of one week to one year

- Short-term load forecasting with the lead time of 24 to 168 hours

- Very short-term load forecasting with the lead time shorter than one day

Short term load forecasting plays a key role in system operation because the scheduling of the generators must be performed every day and it is necessary task for the power dispatch of generators [8]. It is also resolve the issues regarding to the reliability, security and efficiency of the system. Under prediction of STLF leads to insufficient reserve capacity preparation and, in turn, increases the operating cost by using expensive peaking units. On the other hand, over prediction of STLF leads to the unnecessarily large reserve capacity, which is also related to high operating cost[7].

\section{Solution Methodologies}

Most Frequently used solution methods for the short term load forecasting are Statistical methods, similar day approach, linear and multiple regression, Time series method, State space, Exponential Smoothing, Square Estimation, Box and Jenkins Methods, Kalman Filter and State Estimation. Artificial Intelligent Techniques Includes Artificial Neural Network (ANN),SVM(Support Vector Machine Regression) Adaboost regression , Fuzzy logic, SO(Particle Swarm Optimization),GA(Genetic Algorithm),ACA(Ant Colony 
Algorithm),Support vector machine regression and adaboost regression are the most promising techniques which provides the wide potential research in the STLF area [8]. All though there are more than 70 research papers on STFL by using ANN but the SVM regression overcome the difficulty of the ANN by reducing the time during training the historical data. Hybrid Techniques consist of the Fuzzy and ANN, Adaptive neural fuzzy interface system, LS-SVM Optimized by Bacterial Colony Chemotax is Algorithm and GA based SVM.Most frequently used methods are Neural Network and its hybrid models but now a days the SVM regression and other machine learning algorithm replacing neural network having advantage of the low computational time and provides better model by introducing the kernels. So far by using the above mentioned techniques we can predict the future load from the historical load data and we can save a lot of economy by this and also this accuracy can be increased by introducing other features along with the past data of load which has strong correlation with electric load. Solution methods for the short term load forecasting are divided into the following categories.

- Conventional Methods

- Artificial Intelligence Methods

- Conventional Methods

These are the techniques used in STLF Different STLF models are developed by using these methods and techniques. Models are developed by showing the input and output relationships between load and its relative factors after training the historical data[9].

\section{Conventional Methods}

Following conventional method are used in short term load forecasting

\section{Similar-day approach}

The method is used to forecast the load a day, hour or month by searching the similar characteristics day, hour or month in the historical load data. The similar day characteristic include the temperature, date day of week and other influential factors.

\section{Regression methods}

Regression is the one of most widely used statistical techniques for the electric load forecasting. Regression methods are usually used to model the relationship of load consumption and other factors such as weather, day type, and customer class.

\section{Time series}

Time series methods are based on the assumption that the data have an internal structure such as autocorrelation trend or seasonal variation. Most widely used models are ARMA (autoregressive moving average), ARIMA (autoregressive integrated moving average), ARMAX (autoregressive moving average with exogenous variables), and ARIMAX (autoregressive integrated moving average with exogenous variables)

\section{Expert System}

Expert system uses the rules and procedure developed by the human experts in to software for the prediction of electric load. These methods are also called heuristic techniques[10].

\section{Artificial Intelligence Methods}

Besides the conventional techniques used for load forecasting problems, there are some artificial intelligence methods which are used for the modelling of short term load forecasting some of the most frequently used artificial intelligence tools used are.

- Artificial Neural Network (ANN)

- Support Vector Machine (SVM)

- Fuzzy logic

- Practical Swarm Optimization(PSO)

- Genetic Algorithm(GA)

- Ant Colony Algorithm(ACA) 


\title{
I. Artificial Neural Network
}

Artificial Neural Network technique is extracted from the human mind that the neurons of the human mind are trained from the experience and one these are trained the made the decision on the bases of that particular experience [10]. ANN based techniques are frequently used in many engineering applications and it is also one of the popular techniques used in the modelling of load forecasting problems. In load forecasting problems, a model is trained on the basis of past historical data weights are calculated and then the model is test on the same featured data used for training.

\section{Support Vector Machine}

Support Vector Machines (SVMs) a recent powerful technique for solving classification and regression problems. This approach was originated from Vapnik's statistical learning theory. Unlike neural networks which try to define complex functions of the input feature space, support vector machines perform a nonlinear mapping (by using so-called kernel functions) of the data into a high dimensional feature space. Then support vector machines use simple linear functions to create linear decision boundaries in the new space. The problem of choosing architecture for a neural network is replaced here by the problem of choosing a suitable kernel function [11].

\section{Fuzzy logic}

Fuzzy logic is also one of the frequently used techniques in engineering problems. It consists of the models which are based on predefined knowledge it is also used in the forecasting of eclectic load.

\section{Particle Swarm Optimization}

In particle swarm optimization the idea was originated from the flying of swarm's i.e. how they follow the leaders and move towards their destination. PSO base techniques are also used in the load forecasting problems.

\section{Genetic algorithm}

One of the most meritorious and efficient artificial intelligence tools is GA, which is used in many engineering application with great accuracy and efficiency. The researcher of the power system planning also used GA in the load forecasting problem.GA is based on the concept of human chromosomes, inheritance and other properties relating to the genetic of the human.

\section{Hybrid Techniques}

The last class of the load forecasting solution methodology is the hybrid technique which consists of the combination of one or more conventional or artificial intelligence techniques. Some of the hybrid techniques are discussed below.

\author{
i. $\quad$ Fuzzy and ANN \\ ii. Adaptive neural fuzzy interface system \\ iii. LS-SVM Optimized by Bacterial Colony Chemo taxis Algorithm \\ iv. GA based SVM
}

\section{Conclusions}

In early seventies the statistical methods were mostly used like ARMA,box and jinkin method, in nineties artificial intelligent techniques are which can predict the Short Term Load with greater accuracy and in less computation time the most dominant of these method are ANN, Fuzzy logic but with the development of the Novel Neural network Like SVM and Adaboost, at the end of nineties which provides the path way to the researcher and future direction in this area. At the end is summarized from the review of the last 50 year literature that the accuracy of the STLF can be increased by introducing the extra features like weather factors, Peak day, Holidays in the model.

\section{References}

[1] Yuan Ningl, Yufeng Liu Qiang Ji "Bayesian - BP Neural Network Based Short-term Load Forecasting for Power System" 3rd International Conference on Advanced Computer Theory and Engineering(1CACTE) 2010.

[2] Zohreh Souzanchi-K, Hadi Fanaee-T, Mahdi Yaghoubi , Mohammad-R. Akbarzadeh- T “A Multi Adaptive Neuro Fuzzy Inference System for Short Term Load Forecasting by using Previous Day Features" International Conference on Electronics and Information Engineering (ICEIE 2010)

[3] s. Hassnain, A. Asar, F. Mahmood "Performance of STLF Model from the PSO,Time Series and Regression Perspectives" Proceedings of International Joint Conference on Neural Networks, Atlanta, Georgia, USA, June 14-19, 2009 
[4] XinMA, Hong-xiao WU "Power System Short-term Load Forecasting Based on Cooperative Co-evolutionary Immune Network Model" 2nd International Conference on Education Technology and Computer (ICETC)2010.

[5] Yan Wang, Yuanwei Jing, Weilun Zhao, Yan-e Mao"Dynamic Neural Network Based Genetic Algorithm Optimizing for Short Term Load Forecasting"IEEE 2010.

[6] Amit Jain, E. Srinivas, Rasmimayee Rauta "Short Term Load Forecasting using Fuzzy Adaptive Inference and Similarity" IEEE. 2009

[7] Zhi-biao Shi, Yang Li Tao Yu "Short-Term Load Forecasting Based on LS-SVM Optimized by Bacterial Colony Chemotaxis Algorithm" International Conference on Information and Multimedia Technology.2009.

[8] V. H. Hinojosa, Member, IEEE, and A. Hoese "Short-Term Load Forecasting Using Fuzzy InductiveReasoning and Evolutionary Algorithms" IEEE TRANSACTIONS ON POWER SYSTEMS, VOL. 25, NO. 1, FEBRUARY 2010

[9] Wei Sun "A Novel Hybrid GA Based SVM Short Term Load Forecasting Model” Second International Symposium on Knowledge Acquisition and Modeling 2009

[10] A. R. Koushki1, M. Nosrati Maralloo1, C. Lucas2, A. Kalhor2 “Application of Neuro-Fuzzy models In Short Term Electricity Load Forecast" Proceedings of the 14th International CSI Computer Conference (CSICC'09)

[11] LI Jian, JIANG Zhen-huan "Using Least Squares Support Vector Machines in Short-term Electrical Load Forecasting" International Conference on Management Science \& Engineering (16th) September 14-16, 2009 